\title{
LA DOCENCIA AL AIRE LIBRE \\ EN EDUCACIÓN SECUNDARIA \\ EL CURRÍCULO EN EL ENTORNO
}

\section{OPEN-AIR TEACHING IN SECONDARY EDUCATION \\ CURRICULA AROUND}

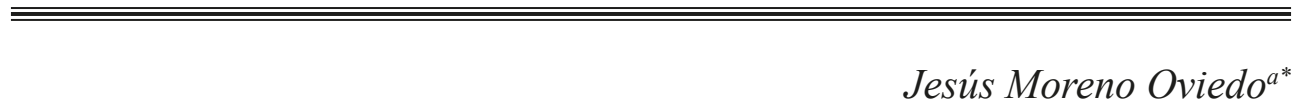

Fechas de recepción y aceptación: 16 de marzo de 2021 y 28 de junio de 2021

DOI: https://doi.org/10.46583/edetania_2021.60.842

Resumen: La educación ha sido afectada en casi todos sus aspectos por la actual pandemia de la COVID-19. Las restricciones impuestas en los centros educativos han obligado a cambiar la rutina de los procesos de enseñanza-aprendizaje y una posibilidad ha sido recuperar como alternativa la actividad educativa fuera del centro. Se ha iniciado así, en el IES Isla de León de San Fernando (Cádiz), un camino en el que se ha puesto en marcha una propuesta compuesta de varios aspectos para afrontar este tipo de enseñanza: el trabajo previo de elección de lugares, la elaboración de actividades, el planteamiento de la salida, la metodología utilizada y los objetivos, contenidos y criterios de evaluación que se van a trabajar. Todos estos elementos, previamente citados, son herramientas que han sido utilizadas para descubrir el currículo en cualquier lugar de la vida real, más allá del encorsetamiento de un libro de texto. El resultado obtenido ha sido satisfactorio en aquellas unidades didácticas escogidas y el alumnado ha mostrado su satisfacción, tanto por el grado de atención mostrado como por el desahogo que supone romper con la monotonía del aula y salir del centro.

Palabras clave: educación, pandemia, enseñanza fuera del centro, salida, metodología.

a IES Isla de León, San Fernando (Cádiz).

* Correspondencia: IES Isla de León, San Fernando. Carretera Carraca, s/n. 11100 San Fernando (Cádiz). España.

E-mail: semorovi883@gmail.com 
Abstract: Education has been affected in almost all its aspects by the current COVID-19 pandemic. The restrictions imposed on educational activities have forced a change in the routine of the teaching-learning processes and one possibility has been to recover educational activities outside of the center. Thus, a path has begun in Isla de Leon high school (San Fernando-Cadiz) in which, a proposal has been launched composed of several aspects to face this type of teaching: the previous work of choosing places, the development of activities, the approach to the excursion, the methodology used and the objectives, contents and evaluation criteria to be worked on. All the elements, previously outlined, are tools that have been used to discover the curriculum in any corner of the real world beyond the confinement of a textbook. The result obtained has been satisfactory in those units chosen and students have shown their satisfaction both by the degree of attention shown and by the relief involved in breaking with the monotony of the classroom and go out of the centre.

Keywords: education, pandemic, open-air teaching, outdoors class, methodology.

\section{JUSTIFICACIÓN Y FUNDAMENTACIÓN}

La declaración por parte de la Organización Mundial de la Salud (OMS) de la pandemia de COVID-19 el 11 de marzo de 2020 ha trastocado el mundo que conocíamos y, por ende, el modo de enseñar y aprender en los centros educativos. Las distintas administraciones educativas han tratado de afrontar esta situación: a nivel nacional, a partir del Real Decreto Ley del 29 de septiembre de 2020, por el que se adoptan medidas urgentes en el ámbito de la educación no universitaria y, concretamente en Andalucía, a partir de la circular de 3 de septiembre de 2020, relativa a medidas de flexibilización curricular y organizativa para el curso 2020-2021. Ambos textos ponen el énfasis en la globalización del currículo para adquirir las competencias clave. Como consecuencia de esto, se planteó la posibilidad de cambiar el escenario de enseñanza y aprendizaje tradicional en un aula y buscar otras opciones, entre las cuales vuelve a cobrar importancia la enseñanza al aire libre. Ya en los primeros años del siglo xx, la Waldschule de Charlottenburg en Berlín (1905), la Open Air School en Londres (1908), la Fresh Air School en Estados Unidos (1911), la École a pleine air en Lyon (1914), así como los trabajos de la Institución Libre de Enseñanza en España en los años diez y veinte del siglo pasado, abrieron el camino a este tipo de docencia, que se orientaba, además de hacia una finalidad de profilaxis, para así prevenir las enfermedades de la infancia, hacia una visión 
rompedora de la docencia, pues trasladaba la enseñanza fuera de los muros de las escuelas y proponía un aprendizaje vivencial y por descubrimiento. En palabras del fundador de esta institución, Giner de los Ríos (1884): “en la escuela como conjunto de espacios, deben primar los espacios abiertos sobre las salas cerradas, manifiesta además su rechazo a los centros monumentales que al final opina, dejaban de ser educativos" (laescueladelarepublica.es).

Un ejemplo de lo anteriormente expuesto fue la llamada "escola del bosc" de Barcelona (1914-1923), creada por la Mancomunidad de Cataluña y que “pretendió modernizar y universalizar la enseñanza desde los primeros años hasta la Universidad o las escuelas de capacitación profesional (El Nacional. cat, 8 de mayo de 2018).

La hipótesis de trabajo inicial se basó en comprobar la viabilidad de este tipo de enseñanza en la impartición de un grupo de materias y el resultado que esta iba a tener sobre nuestro alumnado, partiendo de la base de que estas experiencias encierran una metodología innovadora, basada en un aprendizaje significativo y, en las circunstancias actuales, ofrecen además una metodología de inclusión escolar, pues rompe con la monotonía del aula y estimula al alumno/a. Esta forma de aprender consigue también que los alumnos/as estén activamente comprometidos en crear su propio proceso de aprendizaje y descubran que su entorno más cercano está lleno de elementos que estudian en el aula. Y, finalmente, si algo desarrolla de manera evidente la docencia al aire libre, es el aprendizaje competencial, pues el alumno ha de utilizar las competencias clave: participa de manera constructiva y eficaz en la vida social, ya que se refuerza la cohesión con la realidad más inmediata (competencia social y cívica), utiliza recursos tecnológicos para la búsqueda de información y resolución de problemas (competencia digital) u organiza su propio aprendizaje, gestionando el tiempo y la información (competencia de aprender a aprender). Como afirma Bernal Martínez (2012), comentando aquellas experiencias de principios del siglo xx: "las escuelas al aire libre ofrecían a los maestros una buena oportunidad de plantear la enseñanza desde nuevas perspectivas metodológicas. Las características específicas de estos centros podían favorecer la puesta en práctica de los principios pedagógicos de la escuela activa. (...) más aire en los pulmones debería significar también más aire en los programas y en los horarios, más libertad en el pensamiento y acción de los niños” (p. 175). 
Toda la anterior experimentación didáctica reseñada se planteó de la mano de las recomendaciones de distintas autoridades educativas en países europeos de nuestro entorno que aconsejaban el uso de espacios al aire libre para la docencia en estos tiempos de pandemia. Así, el Ministerio de Instrucción Pública italiano señala en sus recomendaciones para el curso 2020-2021: "la organización de la actividad educativa y docente debe prever la mejora y el uso de todos los espacios internos y externos siempre que sea posible, y en una medida limitada, en caso de condiciones climáticas favorables, el uso de espacios abiertos" (p. 20).

Este estudio aborda la propuesta que un grupo de docentes del IES Isla de León en San Fernando (Cádiz) implementó a través de un grupo de trabajo, haciendo que los alumnos/as recuperaran los espacios públicos después de meses de aislamiento, compartiendo experiencias a nivel metodológico y organizativo, desarrollando aquellos objetivos, contenidos y criterios que se pudieran amoldar mejor a este tipo de enseñanza, analizando la acogida del alumnado, valorando su validez para los procesos de enseñanza-aprendizaje $\mathrm{y}$, finalmente, extrayendo conclusiones a partir de las evidencias que se desprenden de esta investigación.

Para mostrar las evidencias de esta experiencia educativa, se concibió un blog con la intención de que fuera un lugar de encuentro para compartir las experiencias de cada profesor/a en este tipo de docencia.

Sin embargo, el encorsetamiento del sistema educativo, la poca flexibilidad de los tramos horarios de alumnado y docentes, la elevada ratio en determinados cursos o la necesidad en algunos casos de contar con un segundo profesor/a que supervisara la salida supusieron asumir que había que enfrentarse a un tiempo de experimentación y de ensayo-error. Esta realidad se refleja en el siguiente artículo del periódico El País (6 de octubre de 2020):

El sociólogo Mariano Fernández Enguita cree que en los centros existe también "una enorme inercia" contraria tanto a dar clase al aire libre como a acordar con otras Administraciones el uso de sus instalaciones. "Los centros están acostumbrados a funcionar como recintos. Cierran las puertas una vez que han entrado los alumnos y crean un ambiente protegido, autosuficiente, como un santuario. Hay poca costumbre de salir y colaborar con lo de fuera, cuando en realidad siempre hay motivos sociales o pedagógicos para establecer relaciones. 
Lo que saben hacer el conjunto de los profesores del centro en relación con lo que la sociedad sabe hacer es cada vez más insignificante. Qué mejor que poder recurrir al museo de enfrente, a la fundación de la esquina o al grupo de mujeres de no sé dónde. Pero, aunque hay actitudes distintas, la dominante es encerrarse, simplificar. Es un problema de las organizaciones en general, no solo de los centros educativos.

\section{FIGURA 1}

Alumnado de $3 .^{\circ}$ de ESO realizando un mapa mental de la ciudad en la asignatura de Geografia e Historia

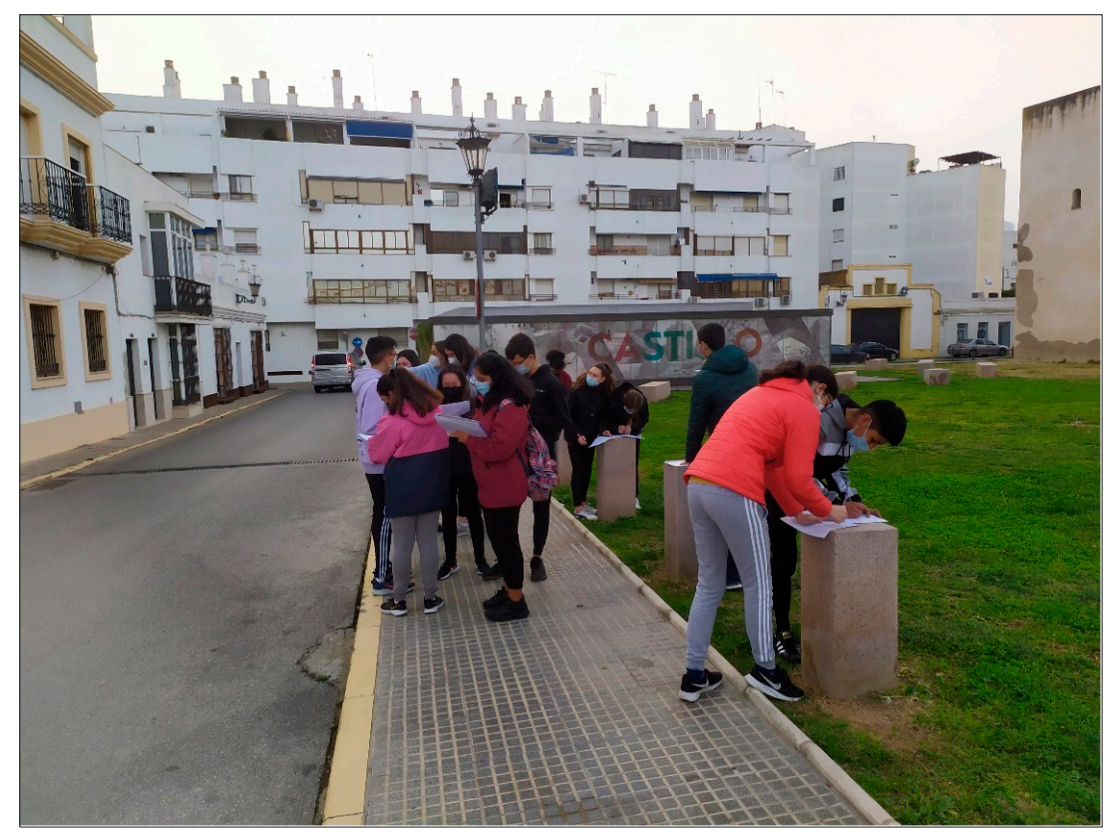

Fuente: www.docencialairelibreisladeleon.blogspot.com

El cambio del escenario docente es, por tanto, algo que va a influir en los procesos de enseñanza-aprendizaje, tanto a nivel organizativo como a la hora de replantear cómo se va a enseñar ese currículo, y para ello se necesitó de un acercamiento teórico a los planteamientos de esta modalidad de docencia. 


\section{CARACTERÍsticas DE LA DOCENCIA AL AIRE LIBRE}

Evidentemente, este tipo de enseñanza encierra unas peculiares características. Recientemente, se han desarrollado estudios y experiencias desde el campo de la educación, tanto para afrontar la situación de pandemia como en contextos no formales, especialmente en educación infantil, que corroboraban nuestra idea previa de aprender haciendo, como afirman García González y Schenetti (2019), "La integración del entorno en la escuela es una idea defendida desde multitud de ámbitos. La experiencia analizada permite la interacción directa del niño con su medio cercano, lo significativo de este tipo de actividad y su necesaria integración en la práctica educativa" (p. 13). La pedagoga Juliet Robertson (2014), especializada en aprendizaje fuera del aula, propone también en su obra Educar fuera del aula algunas pistas en este sentido. Para entender por qué algunas ideas perduran y otras se olvidan, ella plantea estos principios que, a su juicio, se amoldan perfectamente a la docencia al aire libre y que favorecen rutinas en las que se desarrollan las competencias clave. Son los siguientes:

- "Simple: menos es, muchas veces, más. La mayoría de las actividades que se realizan fuera del aula se apoyan en materiales que se hallan in situ y se recurre a la imaginación de los alumnos.

- Inesperado: las interrupciones son frecuentes en las actividades fuera del aula. Por ejemplo, un gato que cruza el patio; descubrir un rodal de setas detrás de un arbusto. Hay que ver estas interrupciones como una parte vital de la clase y dejarse llevar. Aunque no se logre el objetivo original previsto para esa clase, se conseguirá otro.

- Concreto: por lo general, resulta más fácil vincular las experiencias al aire libre con acontecimientos, personas y comunidad. Con frecuencia, se requieren habilidades prácticas que hacen del aprendizaje algo auténtico y real.

- Creíble: parece que trabajar fuera del aula se presta de forma natural a la vida y los intereses de los niños. Estar fuera del aula despierta todos los sentidos, de modo que los niños adquieren una comprensión de las cosas a través de estos.

- Emotivo: aquí entra en juego la capacidad de asombro. No todo el tiempo que se pasa fuera del aula es agradable, pero cuando oyes las expresiones 
de asombro de tus alumnos sabes que se está produciendo una conexión. Aprender es un proceso emocional, además de cognitivo.

- Historias: formar un hilo conductor con las actividades fuera del aula es relativamente fácil. Resulta mucho más complicado hacerlo cuando los niños pasan el día sentados, rellenando páginas de sus cuadernillos de ejercicios. Podemos apoyarnos en las historias para cobrar confianza antes de salir del aula: podemos inventar historias y representarlas. Con frecuencia, las aventuras son los discursos narrativos que se recuerdan" (p. 18).

Tomando como base estos principios, hemos puesto en valor en los procesos de enseñanza-aprendizaje el simple, el concreto, el creíble y el de historias para que estos resulten reforzados frente al tradicional en el aula.

En primer lugar, el simple y el de historias, pues se ha comprobado en actividades evaluables posteriores o en los diarios de aprendizaje del alumnado que este ha retenido mejor lo aprendido en las sesiones al aire libre. Pero también con el concreto y el creíble se ha demostrado que la implicación del alumnado es, en determinados casos, superior, pues se acerca al objeto de aprendizaje y lo percibe como algo aprehensible y útil para su formación.

\section{El trabajo PREVIO}

A lo largo del curso 2020/2021, se ha salido con grupos de todos los niveles tanto en Enseñanza Secundaria Obligatoria como en Bachillerato, y se han buscado lugares que posean elementos conectados con el currículo para hacer la salida más activa y real. En un primer momento, se seleccionaron en el plano de la ciudad las zonas más próximas al instituto y, con un previo trabajo de campo, se escogieron aquellos hitos urbanísticos susceptibles de ser utilizados para llevar a cabo la docencia al aire libre. Ya que el tiempo de una clase es limitado, se acotaron estas zonas, según un cálculo aproximado de quince minutos a pie desde el IES para no disminuir de manera notoria el tiempo de aprendizaje. Del mismo modo, se tuvo en cuenta que este entorno es dinámico y pueden aparecer a lo largo del tiempo nuevos elementos que sumar a los ya elegidos. 
En un segundo momento, hubo que consensuar en los departamentos didácticos, en el grupo de trabajo o en el equipo docente, qué criterios de evaluación de nuestra asignatura podían ser trabajados con los lugares que previamente habíamos escogido (plazas, parques, paseos, jardines, monumentos, etc.). Una vez recopilados, hubo que planificar la salida al exterior.

\section{La salida. Propuestas metodológicas}

El profesor/a elaboraba una serie de preguntas que iban a servir de guía en la salida y que están vinculadas al lugar que se va a visitar y con los contenidos y objetivos que se relacionan con este criterio. Ya en el lugar concreto, el profesor/a hacía una mise en scene del lugar escogido, explicando su porqué. A continuación, los alumnos/as realizaban anotaciones a la explicación que el profesor hacía, descripciones, fotografías o dibujos para, posteriormente en casa, realizar en su cuaderno, diario de aprendizaje o porfolio de clase la tarea, los ejercicios y las actividades propuestos. Otra posibilidad de propuesta fue que el estudiante con un rol más activo lleva a cabo exposiciones, representaciones o aclara dudas a los compañeros/as, siguiendo la metodología de una clase invertida.

En definitiva, la salida al medio promueve como elemento clave la autogestión del aprendizaje. Como afirma M. Cárdenas (2011), "es en este espacio de educación donde el alumnado puede poner en juego todas las competencias que están vinculadas en distintas medidas, a ambos sistemas, tanto el representacional como el perceptual, considerando para ello sus respectivas diferencias metodológicas, así como también las líneas transversales de discusión, la producción de materiales escritos, de materiales visuales y audiovisuales, entre otros" (p. 3).

Uno de los interrogantes más complicados de resolver cuando empezamos a llevar a cabo las clases al aire libre fue la metodología. ¿Cómo desarrollar la clase en un entorno inédito y sin apenas herramientas de un aula convencional? Las primeras experiencias las hemos llevado a cabo en torno a tres líneas fundamentales:

- Como complemento de la clase convencional, sea antes o después, planteando debates de confrontación de ideas, batería de preguntas de repaso 
o lluvia de ideas antes de ver un vídeo, utilizando así el aprendizaje basado en el pensamiento.

- Como role play de determinados contenidos de las asignaturas (pequeñas representaciones teatrales de un momento histórico, formación de una pirámide social o debates de oratoria por grupos), utilizando el aprendizaje cooperativo, en el que el alumnado trabaja en grupos, maximizando su aprendizaje a través de la ayuda mutua.

- Como clase convencional, explicando un determinado contenido cuando este lo encontramos en los alrededores del centro (análisis del sector servicios, de los tipos de transporte, guerra de independencia, Cortes y Constitución de Cádiz en Geografía e Historia o el lenguaje del diseño gráfico en Educación Plástica y audiovisual, entre otros ejemplos).

\section{FIGURA 2}

El uso del libro digital y los dispositivos móviles permite aliviar el traslado de determinados materiales de clase. Grupo de alumnos/as de cuarto de ESO resuelven ejercicios de una tarea, poniendo en práctica la competencia digital

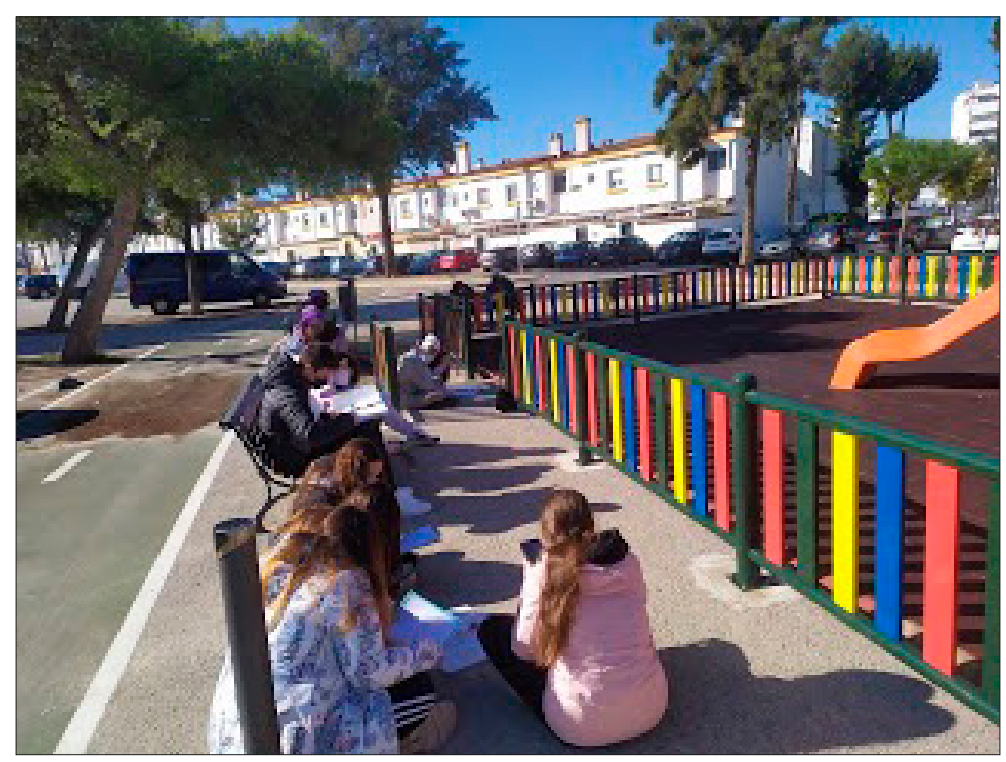

Fuente: www.docencialairelibreisladeleon.blogspot.com 
En el siguiente cuadro se puede observar a modo de ejemplo: cómo determinados criterios de evaluación y contenidos, escogidos en el trabajo previo, pueden ser trabajados en entornos informales de aprendizaje y con alumnado de diferentes etapas educativas.

CUADRO 1

Relaciones curriculares en la enseñanza al aire libre. Algunos ejemplos

\begin{tabular}{|c|c|c|c|}
\hline Criterio de evaluación & Contenidos & Curso & Lugar \\
\hline $\begin{array}{l}\text {-Explicar la organización política y } \\
\text { administrativa de Andalucía, España } \\
\text { y la Unión Europea, analizando el } \\
\text { funcionamiento de las principales ins- } \\
\text { tituciones andaluzas, españolas y de la } \\
\text { Unión Europea. }\end{array}$ & $\begin{array}{l}\text {-La organización polí- } \\
\text { tica de España } \\
\text {-La organización te- } \\
\text { rritorial de España y } \\
\text { Andalucía }\end{array}$ & $3 .^{\circ}$ y $4 .^{\circ} \mathrm{ESO}$ & $\begin{array}{l}\text {-Plaza del Rey } \\
\text { (Ayuntamiento) }\end{array}$ \\
\hline $\begin{array}{l}\text {-Analizar la evolución de los reinos } \\
\text { cristianos y musulmanes, en sus as- } \\
\text { pectos socioeconómicos, políticos y } \\
\text { culturales. } \\
\text {-Entender el proceso de las conquistas } \\
\text { y la repoblación de los reinos cristianos } \\
\text { en la península ibérica y sus relaciones } \\
\text { con Al-Ándalus. }\end{array}$ & $\begin{array}{l}\text {-Los reinos peninsu- } \\
\text { lares }\end{array}$ & 2. ${ }^{\circ} \mathrm{ESO}$ & $\begin{array}{l}\text { Castillo de San Ro- } \\
\text { mualdo }\end{array}$ \\
\hline $\begin{array}{l}\text {-Describir los impactos de los trans- } \\
\text { portes. } \\
\text {-Conocer las características de diversos } \\
\text { tipos de sistemas económicos. }\end{array}$ & $\begin{array}{l}\text {-El transporte } \\
\text {-Actividades econó- } \\
\text { micas }\end{array}$ & $\begin{array}{l}3 .^{\circ} \mathrm{ESO} \\
3 .^{\circ} \mathrm{ESO}\end{array}$ & Estación RENFE \\
\hline $\begin{array}{l}\text {-Reconocer las características de las } \\
\text { ciudades españolas y las formas de } \\
\text { ocupación del espacio urbano, anali- } \\
\text { zando el modelo urbano andaluz y de } \\
\text { ocupación del territorio. }\end{array}$ & -El urbanismo & 2. ${ }^{\circ}$ y $3^{\circ} \mathrm{ESO}$ & $\begin{array}{l}\text {-Recinto de la Mag- } \\
\text { dalena }\end{array}$ \\
\hline $\begin{array}{l}\text {-Señalar en un mapamundi las grandes } \\
\text { áreas urbanas y realizar el comentario, } \\
\text { valorando las características propias de } \\
\text { la red urbana andaluza. }\end{array}$ & -La población & 2. ${ }^{\circ}$ y $3 .^{\circ} \mathrm{ESO}$ & $\begin{array}{l}\text {-Recinto de la Mag- } \\
\text { dalena }\end{array}$ \\
\hline
\end{tabular}




\begin{tabular}{|c|c|c|c|}
\hline Criterio de evaluación & Contenidos & Curso & Lugar \\
\hline $\begin{array}{l}\text {-Explicar las características de los prin- } \\
\text { cipales hechos y procesos históricos de } \\
\text { la península ibérica desde la prehistoria } \\
\text { hasta la desaparición de la monarquía } \\
\text { visigoda, identificando sus causas y } \\
\text { consecuencias de cada etapa histórica. }\end{array}$ & $\begin{array}{l}\text {-Tartessos, indoeuro- } \\
\text { peos y colonizadores } \\
\text { orientales. Hispania } \\
\text { romana: conquista y } \\
\text { romanización de la } \\
\text { península; el legado } \\
\text { cultural romano }\end{array}$ & 2. ${ }^{\circ}$ Bachillerato & $\begin{array}{l}\text {-Recinto de la Mag- } \\
\text { dalena }\end{array}$ \\
\hline $\begin{array}{l}\text {-Explicar las características del "Anti- } \\
\text { guo Régimen" en sus sentidos político, } \\
\text { social y económico. } \\
\text { - Describir las características del nuevo } \\
\text { modelo de Estado, especificando el al- } \\
\text { cance de las reformas promovidas por } \\
\text { los primeros monarcas de la dinastía } \\
\text { borbónica. }\end{array}$ & $\begin{array}{l}\text {-España en el siglo } \\
\text { XVIII } \\
\text {-España en la órbita } \\
\text { francesa. El reformis- } \\
\text { mo de los primeros } \\
\text { Borbones }\end{array}$ & $\begin{array}{l}\text { 4. }{ }^{\circ} \mathrm{ESO} \\
2 .^{\circ} \text { Bachillera- } \\
\text { to. }\end{array}$ & $\begin{array}{l}\text {-Arsenal de la Ca- } \\
\text { rraca }\end{array}$ \\
\hline $\begin{array}{l}\text {-Comprobar el alcance y las limitacio- } \\
\text { nes de los procesos revolucionarios de } \\
\text { la primera mitad del siglo xIx, identi- } \\
\text { ficando la aportación de Andalucía al } \\
\text { establecimiento de un Estado liberal } \\
\text { en España. } \\
\text {-Comentar la labor legisladora de las } \\
\text { Cortes de Cádiz, relacionándola con el } \\
\text { ideario del liberalismo. }\end{array}$ & $\begin{array}{l}\text { - Las Cortes de Cádiz } \\
\text { y la Constitución de } \\
1812\end{array}$ & $\begin{array}{l}4 .^{\circ} \text { ESO } \\
2 .^{\circ} \text { Bachillera- } \\
\text { to. }\end{array}$ & $\begin{array}{l}\text {-Portada de la Iglesia } \\
\text { Mayor de San Pedro } \\
\text { y San Pablo } \\
\text {-Teatro de las Cortes } \\
\text {-Ayuntamiento } \\
\text {-Puente Zuazo }\end{array}$ \\
\hline $\begin{array}{l}\text {-Participar en debates, en los que se } \\
\text { haya recopilado informaciones, por } \\
\text { medio de las tecnologías de la in- } \\
\text { formación y la comunicación, sobre } \\
\text { problemáticas económicas, sociales } \\
\text { y políticas del mundo actual compa- } \\
\text { rándolas con la situación existente en } \\
\text { Andalucía, y realizar estudios de caso, } \\
\text { utilizando para ello las tecnologías } \\
\text { de la información y la comunicación, } \\
\text { sobre la interrelación entre conflictos } \\
\text { sociales y políticos y las diversas for- } \\
\text { mas de discriminación, subrayando las } \\
\text { posibles soluciones y vías de acuerdo } \\
\text { para dichos conflictos. }\end{array}$ & $\begin{array}{l}\text {-Polivalente para cual- } \\
\text { quier contenido }\end{array}$ & Cualquier curso & Plaza de las Vacas \\
\hline
\end{tabular}




\begin{tabular}{|c|l|l|l|}
\hline Criterio de evaluación & \multicolumn{1}{|c|}{ Contenidos } & \multicolumn{1}{c|}{ Curso } & \multicolumn{1}{c|}{ Lugar } \\
\hline $\begin{array}{l}\text {-Utilizar números naturales, enteros, } \\
\text { decimales, fraccionarios y porcentajes } \\
\text { sencillos, sus operaciones y propieda- } \\
\text { des para recoger, transformar e inter- } \\
\text { cambiar información y resolver pro- } \\
\text { blemas relacionados con la vida diaria. }\end{array}$ & & $1 .{ }^{\circ}$ ESO & Patio del IES \\
\hline $\begin{array}{l}\text {-Realizar composiciones creativas } \\
\text { que evidencien las cualidades técnicas } \\
\text { y expresivas del lenguaje del diseño, } \\
\text { adaptándolas a las diferentes áreas, } \\
\text { valorando el trabajo en equipo para la } \\
\text { creación de ideas originales. }\end{array}$ & & & \\
\hline
\end{tabular}

Fuente: elaboración propia

\section{EJEMPLOS DE ACTIVIDADES}

A partir del grupo de trabajo formado en nuestro centro, se están elaborado actividades de las materias de Matemáticas, Lengua y Literatura, Educación Plástica Visual y Audiovisual y Geografía e Historia, siguiendo el siguiente esquema, con la idea de recopilarlos de una manera ordenada para cursos posteriores. He aquí algunas experiencias realizadas.

I.

Departamento: Matemáticas

Asignatura: Matemáticas

Grupo/nivel: $3 .^{\circ}$ ESO E

Metodología: Trabajo cooperativo

Lugar: Castillo de San Romualdo (San Fernando, Cádiz)

\section{Criterios de evaluación trabajados:}

- C1.8. Desarrollar y cultivar las actitudes personales inherentes al quehacer matemático (competencia matemática y competencias básicas en ciencia y tecnología, competencias sociales y cívicas, sentido de la iniciativa y espíritu emprendedor, conciencia y expresiones culturales). 
- C1.9. Superar bloqueos e inseguridades ante la resolución de situaciones desconocidas (aprender a aprender, sentido de la iniciativa y espíritu emprendedor).

- C1.10. Reflexionar sobre las decisiones tomadas, aprendiendo de ello para situaciones similares futuras (aprender a aprender, competencias sociales y cívica, conciencia y expresiones culturales).

- C1.11. Emplear las herramientas tecnológicas adecuadas, de forma autónoma, realizando cálculos numéricos, algebraicos o estadísticos, haciendo representaciones gráficas, recreando situaciones matemáticas mediante simulaciones o analizando con sentido crítico situaciones diversas que ayuden a la comprensión de conceptos matemáticos o a la resolución de problemas (y competencia matemática y competencias en ciencia y tecnología, competencia digital y aprender a aprender).

- C3.2. Utilizar el teorema de Tales y las fórmulas usuales para realizar medidas indirectas de elementos inaccesibles y para obtener medidas de longitudes, de ejemplos tomados de la vida real, representaciones artísticas como pintura o arquitectura, o de la resolución de problemas geométricos (competencia matemática y competencias en ciencia y tecnología y conciencia y expresiones culturales).

Actividad: Midiendo alturas inaccesibles.

\section{Desarrollo de la actividad:}

En el día de hoy hemos llevado a cabo la aplicación del teorema de Tales a situaciones reales de la vida cotidiana, haciendo uso del espectacular contexto de nuestro entorno.

Para ello, el alumnado ha tenido que medir la altura de una de las torres del castillo de San Romualdo, monumento emblemático que se encuentra a escasos segundos del centro.

El método de los espejos consiste en formar dos triángulos y comparar (relacionar) sus lados, gracias a que son semejantes y a la aplicación del resultado mencionado. Por ende, necesitan un espejo que estará en una posición intermedia entre la torre y el alumno. 


\section{II.}

Departamento: Dibujo

Asignatura: EPVA (Educación Plástica Visual y Audiovisual)

Grupo/nivel: $1 .^{\circ}$ ESO A, C, D y F

Metodología: Creativa y expresiva

Lugar: Plaza Cruz de Galicia

Criterios de evaluación trabajados: B1_5.1. Se experimenta con los colores primarios y secundarios estudiando la síntesis aditiva y sustractiva y los colores complementarios (competencia matemática y competencias en ciencia y tecnología).

Actividad: Pintura impresionista al aire libre

Desarrollo de la actividad: Se pintaron paisajes al aire libre con la técnica de la acuarela y partiendo del natural durante dos sesiones con varios grupos, siguiendo los principios de este estilo de pintura. Se recordó al alumnado que los pintores impresionistas realizaban sus obras au plein air, y luego se organizaba un debate entre el alumnado sobre las ventajas e inconvenientes de dicha técnica pictórica.

\section{FIGURA 3}

Desarrollo de una clase de Educación Plástica en la que el alumnado trabaja las texturas de modelos vegetales

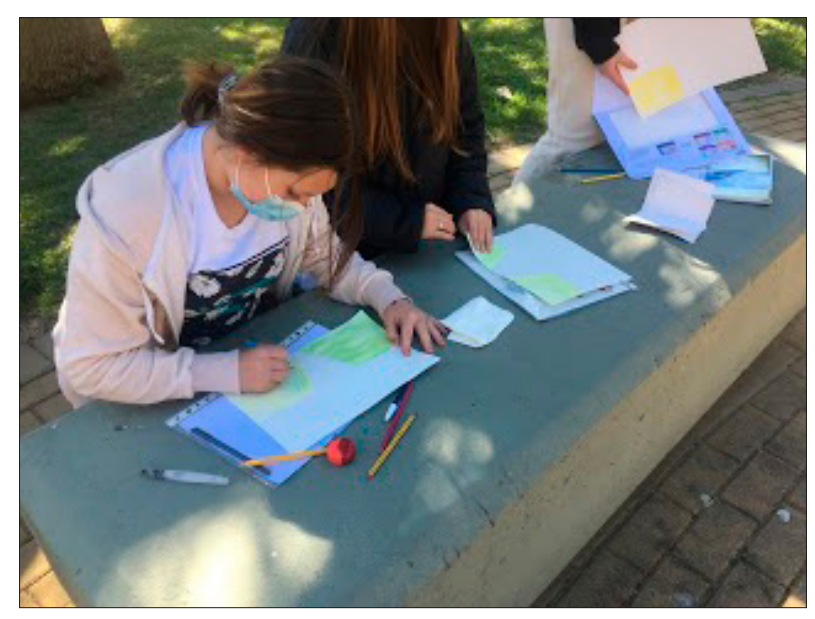

Fuente: www.docencialairelibreisladeleon.blogspot.com 
III.

Departamento: Lengua Castellana y Literatura

Asignatura: Lengua Castellana y Literatura

Grupo/nivel: $3 .^{\circ}$ ESO E

Metodología: Colaborativa

Lugar: Castillo de San Romualdo, zona ajardinada

Actividad: Lectura al aire libre de narrativa contemporánea juvenil

Criterios de evaluación trabajados:

Bloque 2: Comunicación escrita

2. Leer, comprender, interpretar y valorar textos. Aplicar los conocimientos sobre la lengua y las normas del uso lingüístico para solucionar problemas de comprensión y para la composición y la revisión dirigida de los textos adecuados al nivel.

Bloque 4: Educación literaria

1. Leer obras de la literatura española y universal de todos los tiempos y de la literatura juvenil, cercanas a los propios gustos y aficiones, mostrando interés por la lectura (competencia en comunicación lingüística, aprender a aprender, competencias sociales y cívicas y conciencia y expresiones culturales).

2. Favorecer la lectura y comprensión de obras literarias de la literatura española y universal de todos los tiempos y de la literatura juvenil, cercanas a los propios gustos y aficiones, contribuyendo a la formación de la personalidad literaria (competencia en comunicación lingüística, aprender a aprender, competencias sociales y cívicas y conciencia y expresiones culturales).

4. Fomentar el gusto y el hábito por la lectura en todas las vertientes: como fuente de acceso al conocimiento y como instrumento de ocio y diversión que permite explorar mundos diferentes a los nuestros, reales o imaginarios (competencia en comunicación lingüística, aprender a aprender, competencias sociales y cívicas y conciencia y expresiones culturales).

La obra que estamos leyendo en este trimestre, en el curso de $3 .^{\circ}$ ESO E, es Las lágrimas de Shiva, de César Mallorquí. La actividad consiste en leer en voz alta haciendo rondas de lectura entre los propios alumnos; entre ellos mismos también se realizan preguntas de comprensión y de atención a la lectura.

Es importante que los alumnos tengan un modelo de referencia a la hora de la entonación y de pronunciación a la hora de leer. Por este motivo, siempre 
me incluyo en la rueda de lectura e incluso le tomo el turno a alguno si veo que está decayendo en el ritmo. Además de la lectura en voz alta, dedicamos unos minutos de lectura individual en silencio. Posteriormente, ponemos en común lo que hemos leído y se realizan preguntas de comprensión lectora.

Esta actividad está resultando todo un éxito, los alumnos han perdido la vergüenza de leer en espacios abiertos y cada vez su nivel de concentración es mayor cuando leen en silencio.

\section{FIGURA 4}

Clase de Matemáticas al aire libre. Aplicación del teorema de Tales, midiendo una de las torres del castillo de San Romualdo

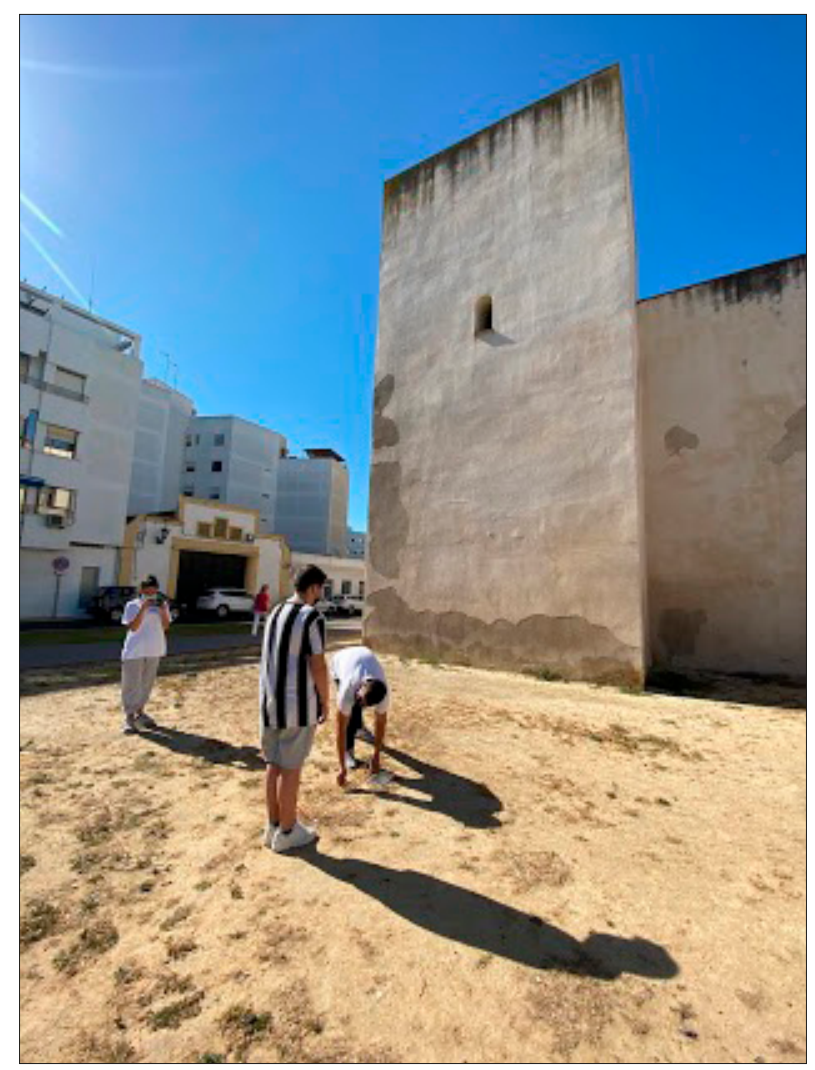

Fuente: www.docencialairelibreisladeleon.blogspot.com 


\section{IV}

Departamento: Geografía e Historia

Asignatura: Historia de España

Grupo/nivel: 2. ${ }^{\circ}$ de Bachillerato A

Metodología: Aprendizaje basado en el pensamiento

Lugar: Plaza de las Vacas

\section{Criterios de evaluación trabajados:}

- HES 5.2. Comentar la labor legisladora de las Cortes de Cádiz, relacionándola con el ideario del liberalismo (competencia en comunicación lingüística, competencias sociales y cívicas).

- HES 6.3. Explicar el proceso constitucional durante el reinado de Isabel II, relacionándolo con las diferentes corrientes ideológicas dentro del liberalismo y su lucha por el poder (competencia en comunicación lingüística, aprender a aprender).

- HES 6.4. Explicar el Sexenio Democrático como periodo de búsqueda de alternativas democráticas a la monarquía isabelina (competencia en comunicación lingüística, aprender a aprender).

- HES 7.1. Explicar el sistema político de la Restauración, distinguiendo su teoría y su funcionamiento real (competencia en comunicación lingüística, aprender a aprender, competencia digital).

Actividad: La evolución del liberalismo en el siglo XIX a través de sus constituciones.

\section{Descripción de la actividad:}

La dificultad que para el alumnado supone tanto entender la evolución política de España en el siglo XIX, como la comprensión de términos tan abstractos relacionados con el liberalismo y las constituciones, me llevó a plantear una actividad en la que, tras leer las características principales de cada constitución del siglo XIX, los alumnos/as pensaban qué cantidad de liberalismo (representado con agua) debía ser vertido a cada uno de los vasos que simbolizaban las constituciones. Posteriormente, al establecerse un debate en la clase sobre el porqué de la dificultad en la implantación del liberalismo en España, los discentes supieron explicar con mayor claridad este proceso histórico. 


\section{FIGURA 5}

Resultado final de un mapa mental que recoge las distintas actividades del sector terciario

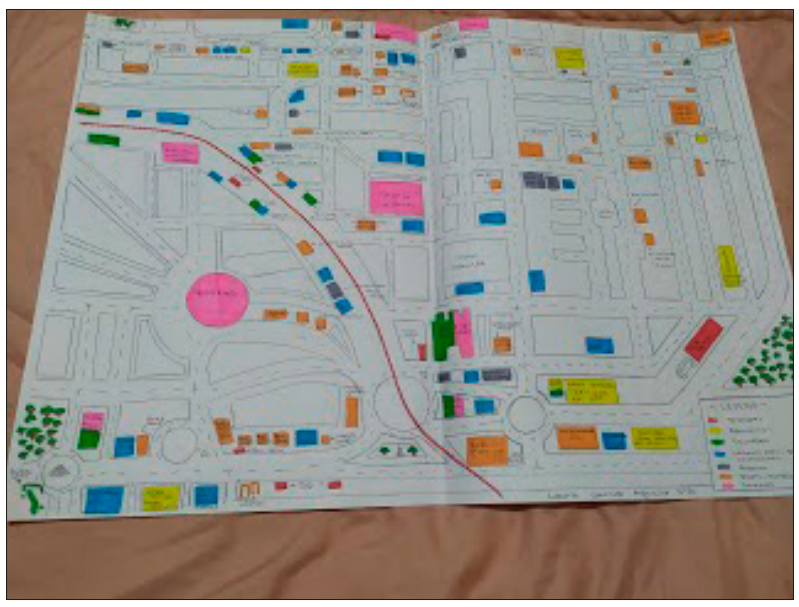

Fuente: www.docencialairelibreisladeleon.blogspot.com

\section{FIGURA 6}

La generalización de la enseñanza sincrónica hizo replantear las sesiones de docencia al aire libre para atender a la totalidad del alumnado del grupo-clase. En la imagen, uso de un dispositivo móvil con trípode para mantener conexión con el resto del alumnado

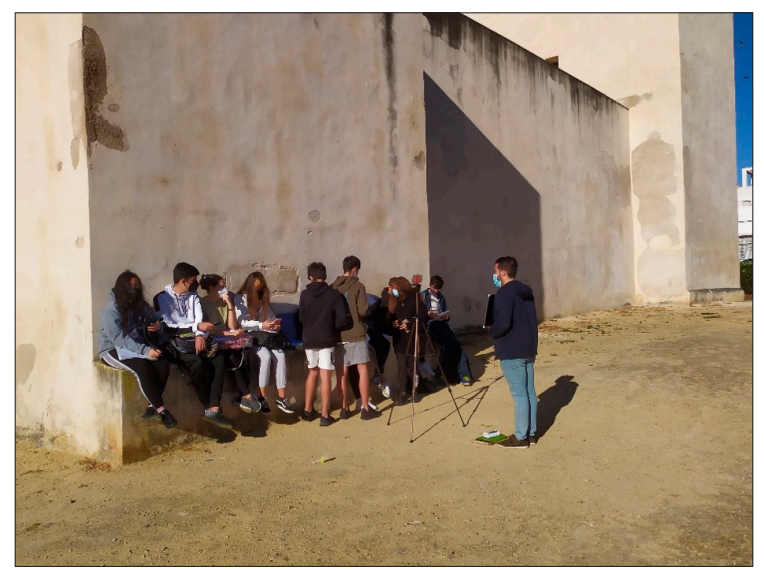

Fuente: www.docencialairelibreisladeleon.blogspot.com 


\section{Resultados del aprendizaje. Conclusiones}

El alumnado, en general, ha demostrado una actitud proactiva hacia las clases al aire libre, y ha aprovechado el tiempo de clase en mayor medida que en el aula ordinaria, colaborando ante una metodología novedosa que descarta las actividades tradicionales (García-Delgado, 2020): “descartar, las actividades estructuradas, cerradas y provistas por los libros de texto que resultan muy limitadas para los complejos procesos de enseñanza-aprendizaje que se desarrollan en estos escenarios. Además, implica repensar los espacios de la escuela para orientarlos hacia estas nuevas perspectivas" (p. 6). Se compensó así la pérdida de tiempo de aprendizaje que se produce en el desplazamiento ante el aumento de la atención y la curiosidad de los alumnos/as. Los resultados del aprendizaje han sido satisfactorios, como se observa en las actividades propuestas y llevadas a cabo que se recogen en este escrito.

Además, hemos observado que este tipo de docencia consigue establecer vínculos entre lo que se aprende en el propio centro y el entorno social en el que este se encuentra, en lugares que también son escuela y donde las posibilidades de aprendizaje se multiplican, como ha demostrado esta propuesta. El alumnado ha descubierto, así, que el currículo no es algo alejado ni de sus inquietudes ni de su vida cotidiana, entendiendo así la educación como algo holístico. Por lo que respecta a los materiales y recursos, la generalización del uso del libro digital y los dispositivos móviles ha permitido aliviar el traslado de determinados materiales de clase. Las encuestas de satisfacción que cumplimentó el alumnado y el profesorado participante en los últimos días de curso reflejan como aspectos positivos de esta experiencia la ruptura con la monotonía del aula-clase, un mayor grado de atención y reflexión por parte del alumnado y una integración mayor del estudiante en su entorno social.

A modo de cierre, se puede afirmar que este proyecto innovador de enseñanza ha cumplido con creces con las expectativas iniciales. Ha demostrado que en los alrededores del centro hay numerosos elementos que se pueden trabajar in situ desde diferentes materias y que similares experiencias, aunque sean en otros niveles educativos o entornos, afirman este carácter innovador, libre, cooperativo y funcional de esta modalidad de enseñanza, que se ve corroborado por los resultados obtenidos a lo largo de estos meses. 


\section{BiBLIOGRAFÍA}

Associazione Nazionale Dirigenti e Alte Professionalità Della Scuola (2020). Vademecum per l'avvio dell anno scolastico. Recuperado de: https:// acortar.link/uP6bKi

Bernal Martínez, J. M. (2012). De las escuelas al aire libre a las aulas de la naturaleza. Áreas. Revista de Ciencias Sociales, 175. Universidad de Murcia.

García Amilburu, M. (2013). En el aula y fuera de ella: educar con la vida. Edetania 43, 83-95.

GarcíA-GonzÁLEz, E. (2020) ¡Urgente, urgente! Re-naturalicemos la escuela en tiempos de pandemia. Revista de Educación Ambiental y Sostenibilidad 2(1), 1.501. DOI: 10.25267//Rev_educ_ambient_sostenibilidad.2020. v2.il.1501

García-GonzÁlez, E. y Schenetti, M. (2019). Las escuelas al aire libre como contexto para el aprendizaje de las ciencias en infantil. El caso de la Scuola nel BoscoVilla Ghigi. Revista Eureka sobre Enseñanza y Divulgación de las Ciencias 16(2), 2.204. DOI: https://orcid.org/0000-0003-0995-9798

La Escuela de la RepúBlica. La Institución Libre de Enseñanza y la Arquitectura escolar. Recuperado de: https://laescueladelarepublica.es/lainstitucion-libre-de-ensenanza-y-la-arquitectura-escolar/

Missiacos CÁrdenas, S. A. (2011). La autogestión del aprendizaje fuera del aula. Univest.

Pons, M. (2018). Se inaugura la escola del bosc, la primera escuela al aire libre. Elnacional.cat. Recuperado de: https://acortar.link/Mo3y9s

RoBERTSON, J. (2014). Educar fuera del aula. Trucos y recursos para ayudar a los docentes a enseñar al aire libre. Biblioteca de innovación educativa. SM, 18

Rodríguez MÉndez, F. J. (2003). Renouvellement architectural et pédagogie de plein air en Espagne (1910-1936), en A.-M. Châtelet (dir.), L'école de plein air. Une expérience pédagogique et architecturale dans l'Europe du XXe siècle. París: Éditions Recherche, 148.

ZAFrA, I. (2020). ¿Por qué no se da clases en los parques? El País (5 octubre de 2020). Recuperado de: https://acortar.link/5NQhSh 